\title{
Indirect Dark Matter search with the ANTARES neutrino telescope
}

\author{
Jean-Pierre Ernenwein ${ }^{* \dagger}$ \\ Université de la Méditerranée, Centre de Physique des Particules de Marseille \\ E-mail: ernenweinecppm.in2p3.fr
}

The ANTARES neutrino telescope, comprising 12 lines of photomultipliers immersed in the Mediterranean Sea at a depth of $2500 \mathrm{~m}$, located $40 \mathrm{~km}$ from the French coast, was completed in June 2008. The ANTARES experiment is based on Cerenkov light detection: the neutrino interaction inside the large volume of matter surrounding the detector produces charged particles, which leads to Cerenkov light emission in the water. A 5-line ANTARES was operational for 10 months during 2007. In December 2007, the telescope grew to 10 lines and the full detector (12 lines) has been operational since June 2008. ANTARES is intended to detect neutrinos, both astrophysical and those originating from self-annihilation of Dark-Matter particles. Within the framework of Supersymmetry the lightest supersymmetric particle, the neutralino, is stable assuming R-parity conservation; in this regime it provides a compelling candidate for the Dark-Matter. In addition, the constrained gravity mediated Supersymmetry (mSugra) scenario allows estimates to be made of the expected rates of neutrinos resulting from neutralino annihilation. At present, the Sun is regarded as the best neutralino-induced neutrino source as it is an efficient and nearby gravitational trap for WIMPs. In this paper we will present the expected sensitivity of ANTARES in this context.

Identification of dark matter 2008

August 18-22, 2008

Stockholm, Sweden

\footnotetext{
${ }^{*}$ Speaker.

${ }^{\dagger}$ For the ANTARES Collaboration
} 


\section{The ANTARES detector: general performances and Dark Matter search context}

ANTARES (Astronomy with a Neutrino Telescope and Abyss environmental RESearch)[1][2][3] is a submarine Cerenkov detector located in the Mediterranean sea, $40 \mathrm{~km}$ off Toulon (France), at a depth of 2475 metres. A surface of $0.06 \mathrm{~km}^{2}$ of deep sea bed is instrumented with 12 lines, each carrying 75 photomultipliers tubes (PMTs) located inside pressure resistant spheres. Three spheres and a titanium cylinder housing the electronics compose a storey. A line is a set of 25 storeys separated by 14.5 metres, for a total height of 450 metres (figure 1). A top buoy and an anchor at the sea bed keep the line vertical. An acoustic positioning measurement set-up provides the PMTs position with a precision better than $10 \mathrm{~cm}$ despite the movements of the line due to water currents.
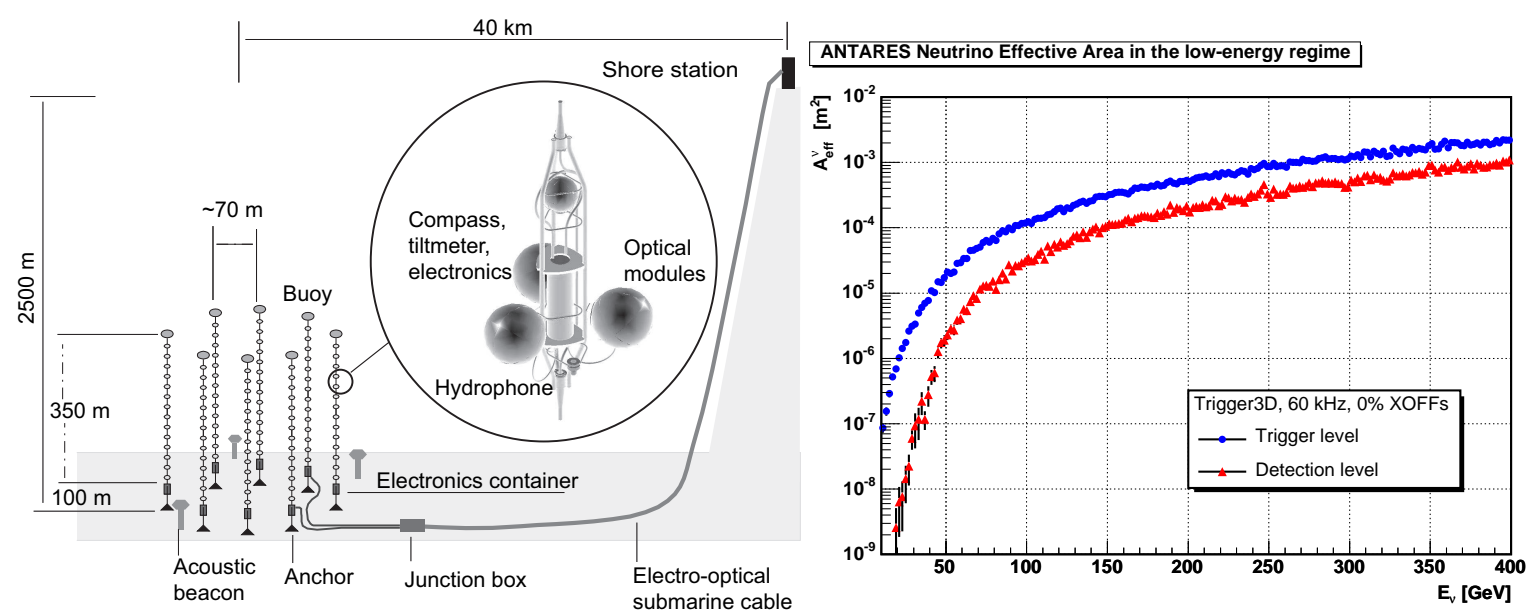

Figure 1: Left: Schematic view of the ANTARES detector, Right: ANTARES Neutrino Effective Area in the low-energy regime

Neutrino Detection principle \& Backgrounds: Depending on neutrino flavour and on current type, the interaction of a neutrino in the vicinity of ANTARES produce an up-going muon and a shower ( $v_{\mu}$ and Charged Current) or only a shower, inducing Cerenkov photon emission along the trajectories of charged particles. PMTs positions and measurements are used to reconstruct the muon track or the shower. Detection of up-going muons is the benchmark for ANTARES : thanks to their long path in matter they allow the detection of neutrino interactions far from the instrumented volume and increase significantly the event rate. However, backgrounds are provided by atmospheric showers: down-going badly reconstructed muons faking up-going muons, and atmospheric neutrinos. In the sea water two other backgrounds generate photons detected by the PMTs: the beta emitter ${ }^{40} \mathrm{~K}$ produces Cherenkov photons, whereas light emitted by bioluminescent organisms mostly leads to single photoelectron production on the PMT photocathode.

Timing \& Angular Resolution: From the time and amplitude of the fired PMT, the muon can be reconstructed using a likelihood fit. The time resolution reachable for single photoelectrons is better than $1.5 \mathrm{~ns}$, and the detection threshold is set at around $1 / 3$ photoelectrons. The time measurement performances, combined with the low scattering and absorption of photons in ANTARES site sea water, allow an angular resolution better than $0.2^{\circ}$ (median) for muon energy above $10 \mathrm{TeV}$. Below $1 \mathrm{TeV}$, the angular resolution is of the order of 1 degree, mainly dominated by kinematics. 
The effective area is the number of detected events per unit of time divided by the incoming particle flux. In the low energy regime, relevant for Dark Matter search, the effective area is shown in figure 1 .

\section{The Dark Matter Context for ANTARES}

Based on several observational evidences, the Dark Matter is known to constitute $22 \%$ of the density of the Universe. Only demonstrated via its gravitational indirect effects, the Dark Matter is still not well understood. Particle physics provides a compelling candidate for the Dark Matter, in the framework of Supersymmetry: in case of R-parity conservation, the lightest particle predicted by Supersymmetry, the neutralino, constitutes a massive weakly interacting particle (WIMP) able to explain the Dark Matter. A relic density of neutralinos is predictable considering an initial production (Big Bang) followed by an exponential decrease due to the self-annihilation of neutralinos. Today, due to the expansion rate of the Universe, this annihilation is not possible anymore, except in massive bodies like the Sun or the Galactic Center, in which WIMPs are trapped and accumulate. The annihilation rate in these bodies is computable in the context of the supersymmetric models, providing, in the Sun case, a promising point-like source of neutrinos for neutrino telescopes like ANTARES. Indeed neutrinos are produced in the annihilation process, with a flux and energy spectrum predictable on the basis of the models.

\section{Neutralino annihilation in the Sun : fluxes and event rates}

The $v_{\mu}+\bar{v}_{\mu}$ flux resulting from neutralino annihilation in the centre of the Sun is displayed on figure 2top and figure 3left. To achieve these computations, the DarkSUSY simulation package [4] was used, accounting for neutrino oscillations in matter and vacuum as well as for neutrino absorption. The constrained phenomenological framework of the minimal Supergravity model (mSUGRA, computations using ISASUGRA[5]) was used, with the following parameter ranges: scalar mass $m_{0}$ in $[0,8000] \mathrm{GeV}$, gaugino mass $m_{1 / 2}$ in $[0,2000] \mathrm{GeV}$, tri-linear scalar coupling $A_{0}$ in $\left[0,3 m_{0}\right]$, sign of the Higgsino mixing parameter: $\mu>0$, ratio of Higgs fields vacuum expectation values $\tan \beta$ in $[0,60], m_{\text {top }}=172.5 \mathrm{GeV}$,

The local Dark Matter halo density (NFW-model) was set to $\rho_{0}=0.3 \mathrm{GeV} / \mathrm{cm}^{3}$.

Figure 2top shows the integrated neutrino flux above $E_{v_{\mu}}=10 \mathrm{GeV}$ in the $\left(m_{0}, m_{1 / 2}\right)$ plane for two ranges of $\tan \beta$. The highest neutrino flux is produced in the so-called "Focus Point" region, in the vicinity of $\left(m_{0}, m_{1 / 2}\right)=(2000,400)$. In this region the large Higgsino component[6] of the neutralino enhances the neutralino capture rate through $Z$-boson exchange, increasing the final neutrino flux, and favours the neutralino annihilation through the $\chi \chi \rightarrow W W / Z Z$ channel, providing neutrinos with an higher energy, well accessible in ANTARES. Figure 3left shows the neutrino flux as a function of the neutralino mass $m_{\chi}$, the colors indicating the compatibility level of the resulting relic density with that measured by WMAP[7].

Figure 2bottom shows the fraction of excludable models in the $\left(m_{0}, m_{1 / 2}\right)$ plane for the ANTARES detector, on the basis of the neutrino detection rate computed according to the flux displayed in the same figure (top), and to the ANTARES effective area (figure 1). These results account for the atmospheric neutrino background remaining in a 3 degree radius search cone around the Sun position, as well as for the misreconstructed atmospheric muon background, assumed to be $10 \%$ of the previous one. The sensitivities are computed using a Feldman Cousins approach[8][9]: for a 
confidence level of $90 \%$, ANTARES needs to detect at least 6.3 neutrinos/3 years to be sensitive to the neutrino flux. On the figure $3 r i g h t$, which shows the detection rate in ANTARES as a function of the neutralino mass $m_{\chi}$, this results of a sensitivity to the models above the horizontal line $\log _{10}\left(v_{\mu}+\bar{v}_{\mu}\right)=0.8$.
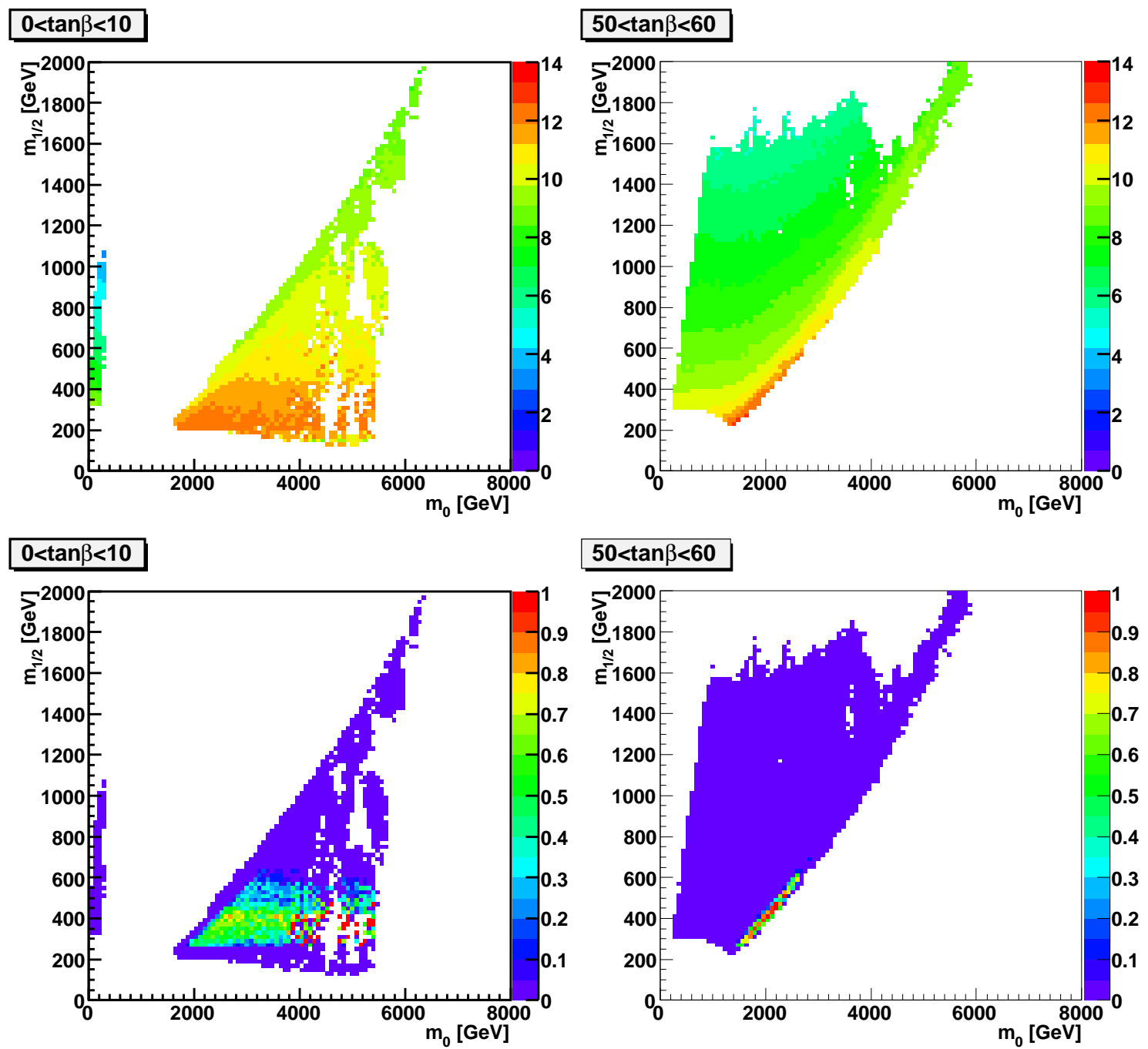

Figure 2: Top: $\log _{10}$ (Integrated flux of neutrinos above $10 \mathrm{GeV} / \mathrm{km}^{2} /$ year). Bottom: fraction of $90 \%$ CL excludable models over the mSugra parameter space: 0 (no sensitvity) to 1 (100\% sensitivity). White regions: models without radiative electroweak symmetry breaking, or $\Omega_{\chi} h^{2}>1$, or already experimentally excluded, or $\chi \neq L S P$.

\section{Conclusion}

The ANTARES detector is fully operational since July 2008. ANTARES will be able to probe the Focus Point region. This feature is common to the direct detection experiments, the spinindependent $\chi p$ cross section which drives these experiments being also enhanced in the Focus Point region[9]. 

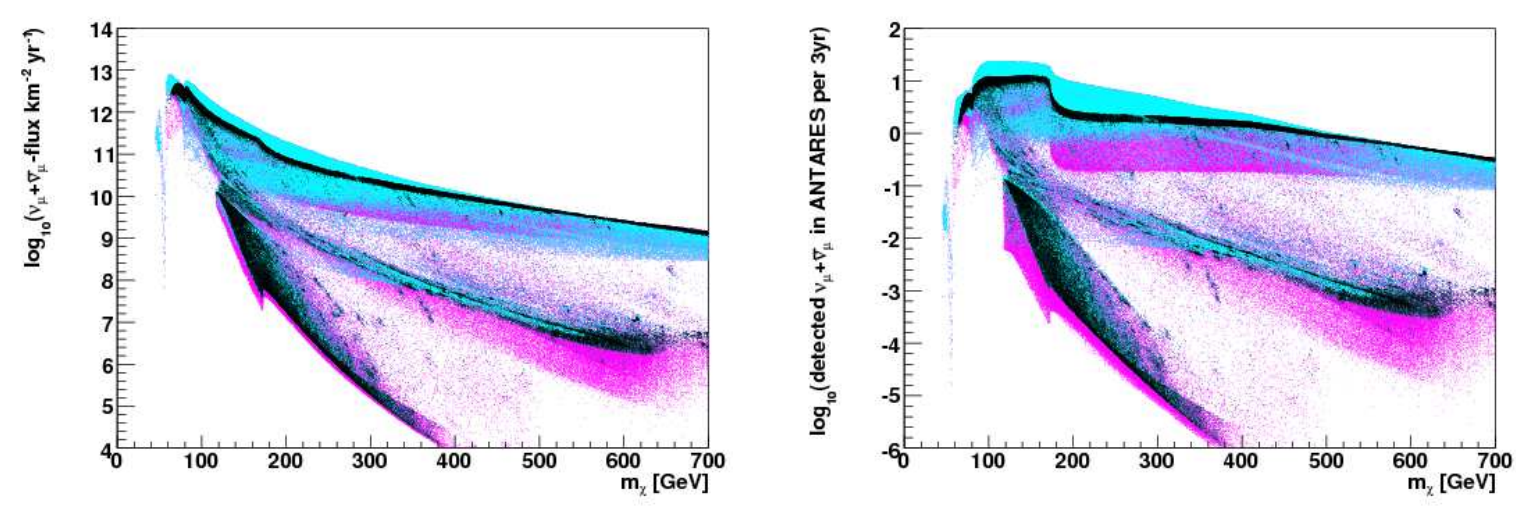

Figure 3: In the mSugra Dark Matter model, considering the LSP annihilation in the Sun, for 3 years of data: Left: neutrino flux for $E_{v_{\mu}}>10 \mathrm{GeV}$, Right: event number expected in ANTARES. Black: $\mid \Omega_{\chi} h^{2}-$ $\Omega_{C D M(W M A P)} h^{2} \mid<2 \sigma$, Cyan: $\Omega_{\chi} h^{2}<\Omega_{C D M(W M A P)} h^{2}-2 \sigma$, Magenta: $\Omega_{C D M(W M A P)} h^{2}+2 \sigma<\Omega_{\chi} h^{2}<1$.

To improve the ANTARES sensitivity for $E_{\mu}<1 \mathrm{TeV}$, which is the domain of interest for Dark Matter searches, efforts are ongoing on the trigger algorithms and on the reconstruction strategies, to increase the effective area.

Nevertheless, although a Cerenkov telescope like ANTARES is mainly optimized for high energies, it is able to give a complementary view of Dark Matter compared to the direct search experiments, at a comparable level of sensitivity.

\section{References}

[1] ANTARES Collaboration, Astropart. Phys. 26 : 314324, 2006

[2] ANTARes Collaboration, Nucl. Instrum. Meth. A581 : 695708, 2007

[3] ANTARES Collaboration, Nucl. Instrum. Meth. A578 : 498509, 2007

[4] P. Gondolo et al., JCAP 0407 (2004) 008

[5] H. Baer et al., arXiv:hep-ph/0312045

[6] Y. Mambrini, E. Nezri, arXiv:hep-ph/0507263

[7] WMAP collaboration, Astrophys. J. 148 (2003) 175

[8] G. Feldman, R. Cousins, Phys. Rev. D 57 (1998) 3873

[9] G. Lim for the ANTARES Collaboration, arXiv:0710.3685v1 [astro-ph] 19 Oct 2007 